\title{
PRINCÍPIO E UTILIZAÇÃO DA TÉCNICA DE EFEITO MIRAGEM DE CONCENTRAÇÃO
}

\author{
João Paulo Campos Trigueiro e Tulio Matencio* \\ Departamento de Química, Universidade Federal de Minas Gerais, CP 702, 31270-901 Belo Horizonte - MG, Brasil \\ Gastón Alvial Moraga \\ Centro de Desenvolvimento de Tecnologia Nuclear, Av. Antônio Carlos 6627, Pampulha, 31270-901 Belo Horizonte - MG, Brasil \\ Christian Lopez \\ Domaine Universitaire, 38402 Saint Martin d'Hères Cedex - France
}

Recebido em 25/4/05; aceito em 24/11/05; publicado na web em 14/6/06

\begin{abstract}
PRINCIPLES AND USE OF THE MIRAGE EFFECT TECHNIQUE - MIRAGE EFFECT OF CONCENTRATION. Abstract: The aim of this work is to present the theoretical and experimental aspects of the mirage effect technique. We are especially interested in the concentration mirage effect, which is a powerful tool in the study of electrochemical reactions that produce ionic movements close to electrodes and to get some fundamental information on mass transport and charge transfer during electrochemical processes. Limitations of this technique are discussed as well as the recent attempts to overcome them.
\end{abstract}

Keywords: in situ technique; refractive index; monitoring of the flux of species.

\section{INTRODUÇÃO}

A eletroquímica é a ciência que trata das relações entre química e eletricidade, descrevendo os fenômenos que ocorrem na interface de um condutor eletrônico, o eletrodo, com um condutor iônico, o eletrólito. Dois processos complementares ocorrem durante uma reação eletroquímica: a transferência de carga elétrica na interface eletrodo/eletrólito e o transporte de massa das espécies redox dentro do eletrólito, que pode acontecer por difusão, convecção ou migração. O conhecimento desses dois processos é de grande importância, pois permite entender a relação entre estrutura e natureza físico-química das espécies que participam da reação, bem como dos fenômenos eletroquímicos ocorridos ${ }^{1}$.

A utilização de fenômenos óticos, como a interferência e a refração para a detecção de perfis de concentração em interfaces do tipo líquido/sólido e líquido/líquido, foi sugerida há mais de 60 anos. Barbero et al. ${ }^{2}$ revelaram que técnicas utilizando a refração da luz para a investigação de gradientes de concentração e a determinação de constantes de difusão já eram publicadas desde 1937. Um extenso artigo de revisão escrito por $\mathrm{Muller}^{3}$ faz citações de estudos de interfaces eletrodo/eletrólito realizados em 1938.

Enquanto os métodos de interferometria não têm recebido reconhecimento significativo nos últimos anos, provavelmente por consequiência de seu complexo aparato experimental, os métodos de refração têm atraído atenção considerável. O aumento de interesse por esses métodos deve-se, principalmente, à disponibilidade de fontes de luz (laser) de baixo custo e alta estabilidade, bem como ao desenvolvimento de detectores de intensidade e posição da luz cada vez mais sensíveis ${ }^{2,4-8}$.

Desenvolvido em 1980 por Bocarra et al. ${ }^{9}$, o Efeito Miragem (EM) tem se destacado entre as técnicas baseadas na refração da luz. Sua aplicabilidade como ferramenta para o estudo das interfaces eletroquímicas foi inicialmente demonstrada em 1987 por Russo et $a l .{ }^{10}$ e por Decker et al. ${ }^{11}$. Trata-se de uma técnica in situ não

*e-mail: tmatencio@ufmg.br destrutiva e de baixo custo, quando comparada a outras utilizadas em eletroquímica.

O objetivo deste artigo é descrever o princípio, as aplicações e os procedimentos experimentais da técnica EM e mais especificamente da técnica EM de concentração, mostrando seu emprego na obtenção de informações fundamentais sobre transporte de massa e transferência de carga durante os processos eletroquímicos.

\section{O PRINCÍPIO DO EFEITO MIRAGEM}

O EM é uma técnica baseada na propriedade da luz de desviar de sua trajetória inicial ao atravessar um meio com índice de refração variável ${ }^{9}$. A Figura 1 mostra o efeito do gradiente de índice de refração sobre a trajetória de um feixe luminoso. A luz, ao incidir sobre um meio contendo um gradiente de índice de refração, é desviada de um ângulo $\theta$ em relação à sua trajetória inicial. Esse desvio ocorre no sentido dos maiores índices de refração.

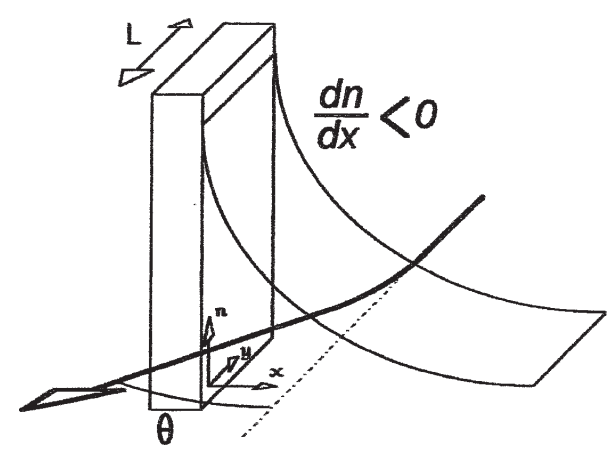

Figura 1. Efeito de um gradiente de índice de refração sobre a trajetória de um feixe luminoso, no caso particular do decréscimo do valor do índice com a distância x. O feixe, ao atravessar uma região de não homogeneidade de índice de refração, é desviado da sua trajetória inicial

A dependência do ângulo de desvio da luz com o gradiente de índice de refração é dada pela seguinte Equação ${ }^{12,13}$ : 
$\theta=\frac{L}{n} \frac{d n}{d x}$

Sendo $\theta$ o ângulo de desvio do feixe em rad, L o comprimento de interação do feixe de luz com a amostra estudada (praticamente a dimensão do eletrodo ao longo do feixe de luz), n o índice de refração do meio não perturbado em contato com a amostra e x o eixo ao longo do qual ocorre a perturbação.

O gradiente do índice de refração pode ser induzido por muitos fatores, como variação de pressão, temperatura e concentração de espécies dissolvidas na solução ${ }^{14,15}$. Se temperatura e concentração forem os dois únicos fatores que variam durante o experimento, o gradiente do índice de refração será expresso por:

$\frac{d n}{d x}=\frac{\partial n}{\partial T} \frac{\partial T}{\partial x}+\frac{\partial n}{\partial C} \frac{\partial C}{\partial x}$

Os termos $\frac{\partial n}{\partial C}$ e $\frac{\partial n}{\partial T}$ expressam a dependência do índice de refração n com a concentração $\mathrm{C}$ e com a temperatura $\mathrm{T}$ do eletrólito, respectivamente, enquanto $\frac{\partial T}{\partial x}$ e $\frac{\partial C}{\partial x}$ expressam os gradientes de temperatura e concentração, respectivamente.

\section{$O$ efeito miragem de temperatura}

Originalmente, o desenvolvimento e os primeiros usos da técnica EM foram feitos a partir de gradientes de temperatura e a técnica era denominada de "Photothermal Deflection Spectroscopy" - PDS, "Photothermal Beam Deflection" PBD ou "Photothermal Deflection Technique" - PDT. Essas técnicas utilizam o feixe de prova para medir mudanças no índice de refração devido, exclusivamente, a variações de temperatura ${ }^{13,16,17}$.

A equação para o cálculo do desvio do feixe de luz, levando-se em conta somente a variação da temperatura, é deduzida das Equações 1 e 2 :

$\theta=\frac{L}{n} \frac{d n}{d T} \frac{d T}{d x}$

sendo que $\mathrm{T}$ corresponde à temperatura do meio em contato com a superfície da amostra.

Boccara et ll $^{9}$ mostraram que a PDS permite medir variações de temperatura de superfície da ordem de $10^{-7}{ }^{\circ} \mathrm{C}$, quando a amostra está mergulhada em um líquido. A PDS está sendo empregada em diferentes tipos de estudos, como caracterização mecânica de metais $^{18}$, medidas de calorimetria ${ }^{19}$, localização de defeitos estruturais em amostras sólidas ${ }^{20,21}$, detecção de mudança de fase em metais $^{22}$, medidas de entalpia de reações químicas ${ }^{23-25}$, monitoramento da difusão de um gás entre duas fases líquidas ${ }^{26}$ e medidas de difusividade térmica de materiais ${ }^{27-35}$.

A PDS é uma técnica muito sensível para o estudo de vários parâmetros de transporte em filmes de semicondutores, como, por ex., difusividade térmica, difusividade eletrônica e velocidade de recombinação de superfície ${ }^{36}$. Mathew et al. ${ }^{37}$ empregaram a PDS para medida da difusividade térmica de diferentes composições de filmes finos de semicondutores amorfos $\mathrm{Ge}_{\mathrm{x}} \mathrm{Se}_{100-\mathrm{x}^{*}}$. Dersch et al. ${ }^{38}$ aplicaram a PDS para medir os calores Peltier e Joule liberados durante a passagem de corrente em um filme fino de silício amorfo (a-Si:H). A PDS foi também empregada no campo da Biologia para caracterização da presença de antígenos em uma matriz biológi$\mathrm{ca}^{39}$ e para medida da atividade fotoquímica em tecidos vivos ${ }^{40,41}$. Existem, na literatura, algumas discussões sobre modelos teóricos relacionados com a $\operatorname{PDS}^{12,42,43}$.

\section{Efeito miragem de concentração}

De modo análogo à temperatura, a equação para cálculo do desvio do feixe de luz, levando-se em consideração somente a variação da concentração, é deduzida das Equações 1 e 2:

$\theta=\frac{L}{n} \frac{d n}{d C} \frac{d C}{d x}$

sendo $\mathrm{C}$ a concentração das espécies dissolvidas na solução. A determinação do termo $\frac{d n}{d C}$ pode ser feita por meio de um refratômetro ${ }^{44}$ ou com a utilização de valores tabelados.

A ocorrência de uma reação na interface eletrodo/eletrólito estabelecerá um gradiente do índice de refração na solução ${ }^{44}$. Como exemplo, pode-se considerar uma espécie $\mathrm{M}^{+}$em solução, susceptível de se reduzir a uma espécie $\mathrm{M}$, insolúvel, depositada sobre um eletrodo. Considerando a Equação (4), podem ser levantadas algumas hipóteses:

1) A variação do índice de refração e da concentração é proporcional $\left(\frac{d n}{d c}>0\right)$

2) Somente uma espécie $\left(\mathrm{M}^{+}\right)$é trocada entre o eletrodo e a solução.

3) O processo redox estudado é isotérmico e isobárico. Somente a variação da concentração da espécie $\mathrm{M}^{+}$pode modificar a trajetória do laser.

As Figuras 2 e 3 mostram como o processo eletroquímico, ocorrido no sistema, influencia o gradiente de concentração e, por sua vez, o ângulo de deflexão.
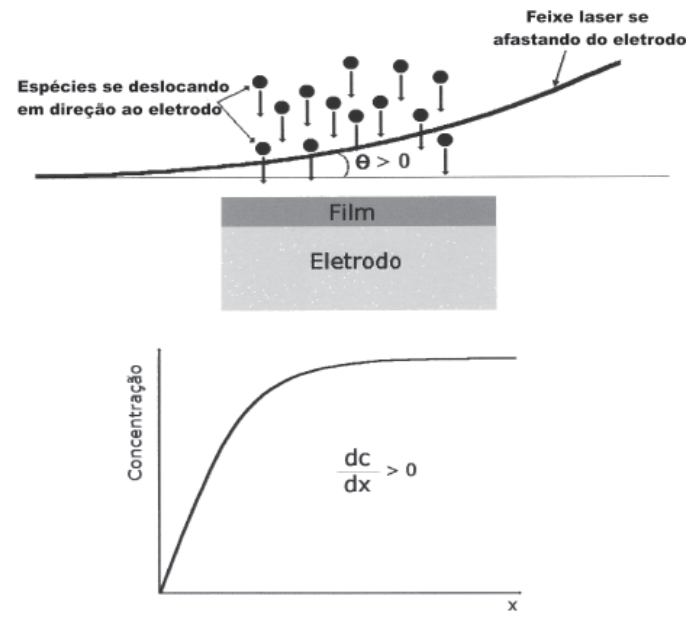

Figura 2. Deflexão do feixe de luz com a variação da concentração do eletrólito (redução, oxidação, etc.): espécies se deslocando em direção ao eletrodo, deflexão positiva

Nessas condições, o processo de redução promove uma diminuição da concentração de espécies solúveis $\mathrm{M}^{+}$na vizinhança do eletrodo (Figura 2). O gradiente de concentração torna-se positivo, acarretando um valor positivo para o ângulo de deflexão da luz. O processo de oxidação, por sua vez, torna o gradiente de concentração negativo, ocasionando um ângulo de deflexão negativo (Figura 3).

Entretanto, as reações redox não são sempre simples assim. Apesar da hipótese de proporcionalidade da variação do índice de refração e da concentração ser válida na maioria das vezes, as duas outras hipóteses não são sempre respeitadas, tornando-se difícil 

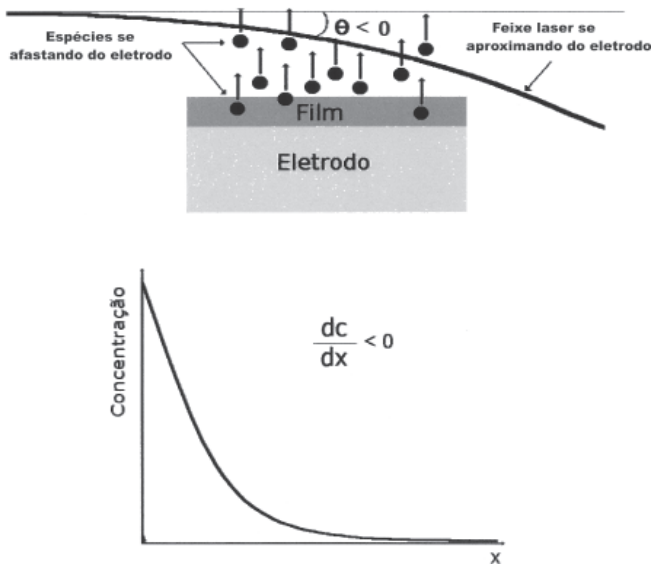

Figura 3. Deflexão do feixe de luz com a variação da concentração do eletrólito (redução, oxidação, etc): espécies se deslocando em direção à solução, deflexão negativa

uma análise quantitativa do sinal miragem. Em algumas situações, os produtos e reagentes podem ser solúveis e provocar um desvio da luz. Barbero et al. ${ }^{2}$ e, posteriormente, Rosolen et al. ${ }^{45}$ mostraram que, nesses casos, a análise tem que considerar coeficientes de difusão e índices de refração diferentes para cada espécie. Outro ponto que pode dificultar a análise do sinal miragem é o fato de as reações eletroquímicas serem acompanhadas por variação de temperatura na superfície do eletrodo e, nesses casos, a variação do índice de refração ser uma função da concentração e da temperatura. Decker et al. ${ }^{16,46}$ mostraram que é possível separar os componentes de temperatura e de concentração do sinal miragem, aplicando perturbações senoidais de corrente de várias frequiências no eletrodo estudado. $\mathrm{O}$ efeito térmico mostrou-se, geralmente, predominante nas altas frequiências e o efeito de concentração nas baixas freqüências. Rosolen et al. ${ }^{45}$ mostraram como o EM pode ser utilizado para medidas calorimétricas e refratométricas nos eletrólitos, discutindo os efeitos térmicos Peltier e Joule, bem como as variações dinâmicas dos perfis de concentração existentes nos eletrólitos. Os autores evidenciaram que, durante a reação eletroquímica, existem trocas de calor relacionadas com a mudança de entropia na interface eletrodo/solução (efeito Peltier) e com a irreversibilidade da reação (efeito Joule). É importante, portanto, estimar a potência térmica gerada durante a reação eletroquímica estudada, a fim de saber se o desvio do feixe laser, durante o experimento, é ou não sensivelmente afetado pelos efeitos Peltier e Joule. Uma potência térmica da ordem de $\mathrm{mW} \mathrm{cm}^{-2}$ apresentará, geralmente, uma participação desprezível no desvio do feixe, em relação ao efeito dos gradientes de concentração.

Em princípio, a dependência do ângulo de desvio da luz com a variação do índice de refração só é aplicável se o diâmetro do feixe de luz for infinitamente pequeno. Entretanto, Rudnicki et al. ${ }^{47}$ mostraram, matematicamente, que um feixe de luz com um diâmetro menor que $100 \mu \mathrm{m}$ pode ser considerado suficientemente pequeno.

Em trabalhos experimentais, geralmente o diâmetro do feixe de luz varia de 20 a $80 \mu \mathrm{m}$. Devido a essa variação, o feixe é focalizado a uma distância de 30 a $100 \mu \mathrm{m}$ em relação ao eletrodo, em uma média experimental ${ }^{48}$. Conseqüentemente, a perturbação do perfil de concentração do eletrólito, induzida por um processo redox localizado no eletrodo, será detectada pela luz após um atraso. Este atraso é devido ao tempo requerido pelas espécies iônicas para se difundirem na solução (cerca de $10 \mathrm{~s}$ para uma espécie com coeficiente de difusão de $\left.10^{-5} \mathrm{~cm}^{2} \mathrm{~s}^{-1}\right)^{49}$. Logo, percebe-se a inviabilidade de uma comparação quantitativa entre o sinal miragem e o sinal de corrente obtido por uma técnica clássica da eletroquímica. Para superar essa dificuldade, alguns modelos que levam em consideração o transporte de massa foram desenvolvidos ${ }^{2,45,47-51}$. Quando a técnica eletroquímica acoplada à técnica EM é a cronoamperometria ou a cronopotenciometria, modelos matemáticos simples com soluções analíticas foram desenvolvidos ${ }^{2,45,47}$. No caso da voltametria cíclica, os modelos são mais complexos devido à não existência de solução analíticas para simulações dos voltamogramas. Vieil et al. ${ }^{49-51}$ desenvolveram uma ferramenta matemática baseada na convolução temporal para simular o desvio do feixe miragem durante uma voltametria cíclica. Nesse tratamento, os autores utilizaram um produto de convolução com uma função de transferência que caracteriza a difusão semi-infinita, predizendo, assim, os fluxos de espécies a uma certa distância do eletrodo de estudo. Mathias ${ }^{48}$ abordou problemas similares através da simulação digital dos fluxos iônicos de migração e difusional em eletrólitos binários.

Pouco tempo após as primeiras publicações sobre a técnica EM e suas aplicações relacionadas com as medidas de gradiente de temperatura, foram feitos os primeiros estudos de medidas de gradiente de concentração ${ }^{41,52}$.

Quando a técnica do EM é acoplada a outras técnicas eletroquímicas usuais, como por ex., voltametria cíclica, cronoamperometria ou cronopotenciometria, se mostra uma ferramenta in situ muito interessante para estudar os processos eletroquímicos que envolvem movimentos iônicos ${ }^{11,14,15,44,45,49,53-67}$. Entretanto, o EM permite monitorar somente o fluxo iônico e não suas quantidades absolutas. No intuito de resolver essa limitação e obter simultaneamente informações sobre movimentos de cargas e massa, alguns grupos de pesquisa acoplaram o EM a uma outra técnica eletroquímica, denominada Microbalança a Cristal de Quartzo (MECQ) $)^{1,61,65-67}$.

\section{O APARATO EXPERIMENTAL DO EFEITO MIRAGEM DE CONCENTRAÇÃO}

A montagem experimental utilizada na técnica EM de concentração é composta basicamente de banco ótico, fonte de luz, cuba eletroquímica, detector de posição, lentes convergentes, plataforma de rotação, plataforma de translação (vertical e horizontal) e sistema de isolamento mecânico. A Figura 4 representa uma montagem que desenvolvemos para trabalhar com a técnica $\mathrm{EM}^{68,69}$.

\section{Banco ótico (1)}

O banco ótico de grande estabilidade é empregado para fixar e alinhar todos os componentes utilizados no experimento.

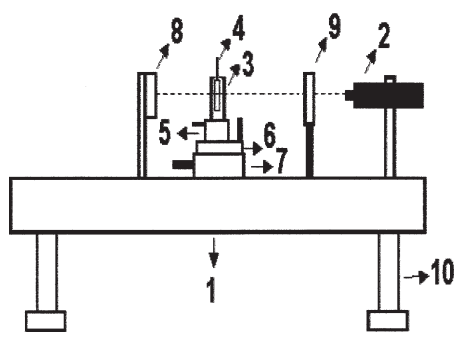

Figura 4. Aparato experimental utilizado para medidas com a técnica EM. 1) banco ótico, 2) laser, 3) cuba eletroquímica, 4) eletrodo de trabalho, 5) plataforma de rotação, 6) plataforma de translação vertical, 7) plataforma de translação horizontal, 8) detector de posição, 9) lente convergente, 10) amortecedor pneumático 


\section{Fonte de luz (2)}

Os lasers são muito utilizados em instrumentação analítica por serem extremamente monocromáticos e conservarem coerência através de uma distância relativamente grande. O termo laser é um acrônimo para "light amplification by stimulated emission of radiation". Em consequiência de suas características de amplificadores de luz, os lasers produzem feixes de radiação extremamente intensos e espacialmente estreitos (alguns centésimos de micrômetro). $\mathrm{O}$ processo de emissão estimulada produz um feixe de radiação altamente monocromático (bandas de larguras de 0,01 nm) e extremamente coerente. Em virtude dessas propriedades únicas, os lasers tornaram-se fontes importantes para uso nas regiões do espectro ultravioleta, visível e infravermelho. O laser de hélio/ neônio, 632,8 nm, é o mais largamente empregado, devido ao perfil gaussiano de sua intensidade, o que assegura sua localização principalmente no centro do feixe. A potência do laser utilizado gira em torno de $2 \mathrm{~mW}^{70,71}$.

\section{Cuba eletroquímica (3)}

A cuba eletroquímica, utilizada para acomodar o eletrólito e os eletrodos, é feita com um material que possui uma transmissão óptica entre 95 e $98 \%$ na região de 400-800 nm. É importante mencionar que a cuba deve possuir janelas perfeitamente normais à direção do feixe minimizando, dessa forma, perdas de radiação por reflexão nas paredes. Impressões digitais, gordura ou outros depósitos nas paredes alteram significativamente as características de transmissão de uma célula. Assim, uma limpeza cuidadosa é imprescindível. Erickson et al. ${ }^{72}$ sugerem uma seqüência de procedimentos para se fazer uma limpeza adequada nas cubas.

\section{Eletrodo de trabalho (4)}

No eletrodo de trabalho são aplicados sinais de potencial gerados por um potenciostato (em geral rampa linear, no caso de uma voltametria cíclica, e pulso no caso de uma cronoamperometria).

\section{Plataforma de rotação (5)}

A plataforma de rotação permite uma rotação micrométrica da cuba eletroquímica em relação ao feixe de laser. É utilizada para garantir o paralelismo da amostra e do feixe de laser. O alinhamento é feito utilizando-se a autocolimação do feixe, ou seja, superpondo-se os feixes incidente e refletido pelas janelas de vidro da cuba. Uma vez encontrado o posicionamento correto, fixa-se essa posição.

\section{Plataforma de translação $(6,7)$}

As plataformas de translação permitem o deslocamento micrométrico da cuba eletroquímica nas direções vertical e horizontal. O deslocamento vertical permite escolher a parte da amostra que será estudada e o deslocamento horizontal permite fazer a medida óptica em regiões diferentes da camada de difusão.

\section{Detector de posição (8)}

O detector de posição, constituído por foto-células de silício sensíveis à luz, permite quantificar a deflexão do feixe laser, gerando um potencial proporcional à superfície iluminada. Para compensar eventuais flutuações do feixe laser, um circuito eletrônico simples realiza uma subtração dos sinais gerados (normalizado por uma soma). O resultado dessa operação é um sinal proporcional à deflexão do feixe laser, o qual depois de um tratamento matemático simples (que será comentado no tópico curva calibração) se transforma no sinal de desvio angular (sinal miragem).

\section{Lente convergente (9)}

A lente convergente desempenha um papel importante na sensibilidade da medida. Colocada entre o laser e a cuba eletroquímica, permite a convergência do feixe perto do eletrodo de trabalho. $\mathrm{O}$ diâmetro do feixe no local é de aproximadamente $32 \mu \mathrm{m}$, condição suficiente para utilização das Equações (1) a (4) ${ }^{47}$. O alinhamento da lente é feito seguindo-se o método interferométrico proposto por Oliveira et al..$^{73}$

\section{Sistema de isolamento mecânico (10)}

Na técnica EM, vibrações e trepidações externas podem interferir significativamente na medida do ângulo de desvio do feixe de laser. Desta maneira, é de fundamental importância que o sistema experimental da técnica seja isolado de perturbações mecânicas externas, para garantia de estabilidade e confiabilidade nas medidas. Na montagem que desenvolvemos, o isolamento mecânico é realizado por quatro colunas, contendo amortecedores pneumáticos.

\section{Estimativa do custo da montagem}

A Tabela 1 apresenta a estimativa dos custos dos equipamentos necessários à montagem do aparato experimental do EM de concentração. Para a realização dos experimentos necessita-se também de um potenciostato/galvanostato e de um computador para aquisição e tratamento dos resultados.

Tabela 1. Estimativa dos custos da montagem do EM de concentração

\begin{tabular}{lr}
\hline Equipamento & Preço em US \$ \\
\hline Banco ótico com amortecedores pneumáticos & 5000,00 \\
HeNe Laser, 2 mW, 633 nm com suporte & 800,00 \\
Lente convergente com suporte & 100,00 \\
$\begin{array}{l}\text { Plataforma de rotação com deslocamento } \\
\text { micrométrico }\end{array}$ & 900,00 \\
$\begin{array}{l}\text { Plataforma de translação horizontal } \\
\text { com deslocamento micrométrico }\end{array}$ & 500,00 \\
$\begin{array}{l}\text { Plataforma de translação vertical com } \\
\text { deslocamento micrométrico }\end{array}$ & 500,00 \\
$\begin{array}{l}\text { Célula eletroquímica (cuba e eletrodos) } \\
\text { Foto-célula fotovoltáica de silício e }\end{array}$ & 150,00 \\
circuito eletrônico & 100,00 \\
\hline
\end{tabular}

\section{CURVA DE CALIBRAÇÃO DO EFEITO MIRAGEM DE CONCENTRAÇÃO}

É necessário estabelecer uma curva de calibração relacionando o sinal elétrico, fornecido pelo circuito eletrônico conectado ao detector, e a posição da superfície iluminada pelo feixe.

A Figura 5 mostra uma representação geométrica do feixe laser após passar pela lente convergente, em nossa montagem experimental.

A distância entre o detector e o centro do eletrodo de trabalho é escolhida de forma a otimizar o sinal fornecido pela foto-célula de silício. Geralmente, os melhores resultados são obtidos quando 


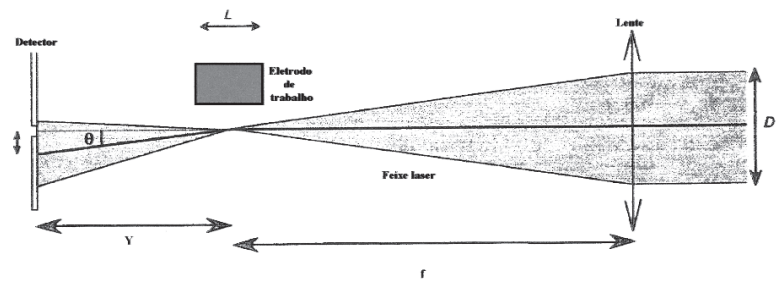

Figura 5. Representação geométrica do feixe laser após passar pela lente convergente. $\Delta d$ : distância entre o centro do feixe e o centro do detector; $\theta$ : ângulo de desvio do feixe; $D$ : diâmetro do feixe laser $(2 \mathrm{~mm})$; $L$ : comprimento da superfície do eletrodo de trabalho $(5 \mathrm{~mm})$; $f$ : distância focal da lente $(80$ mm); Y: distância entre o detector e o centro do eletrodo de trabalho

a área de iluminação do detector é da grandeza da área de uma das superfícies ativas da foto-célula de silício, permitindo o aproveitamento máximo da capacidade do detector. Considerando que $\theta<<$ 1 , o ângulo pode ser aproximado por sua tangente. Desta maneira tem-se a seguinte relação:

$\theta \approx \operatorname{tg}(\theta)=\frac{\Delta d}{Y}$

A Figura 6 representa uma curva de calibração obtida a partir do deslocamento perpendicular do detector em relação ao feixe laser.

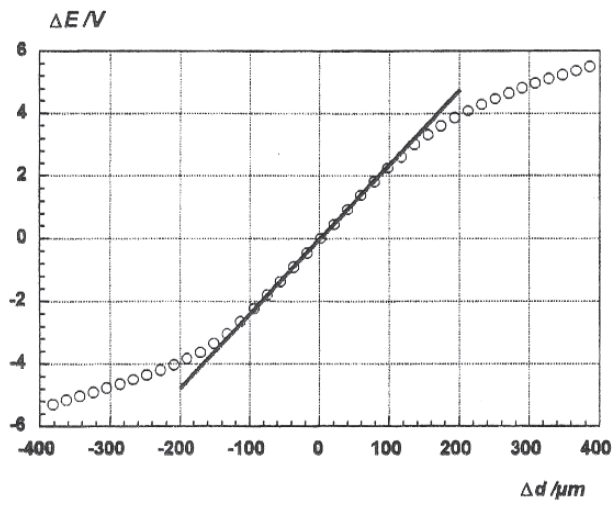

Figura 6. Curva de calibração do EM obtida em nossos experimentos

$\mathrm{O}$ sinal elétrico $\Delta \mathrm{E}$ fornecido pelo circuito eletrônico conectado ao detector é registrado em função do deslocamento $\Delta \mathrm{d}$ do detector. Observa-se que para os deslocamentos situados entre -100 e +100 $\mu \mathrm{m}$ existe uma relação linear entre $\Delta \mathrm{E}$ e $\Delta \mathrm{d}$. Pode-se então expressar, nesta faixa de deslocamento a partir da relação (5) e da curva de calibração, a expressão que relaciona ângulo de desvio e sinal elétrico:

$\frac{\theta}{\Delta E}=0.98 \mathrm{rad} V^{-1}$

O valor deste ângulo, entretanto, precisa ser corrigido para considerar o desvio sofrido pelo feixe laser ao atravessar a parede da célula eletroquímica. A Figura 7 mostra o desvio sofrido pelo feixe laser durante sua passagem pela cuba eletroquímica.

Levando-se em conta a ocorrência do desvio adicional de $\theta$, deve-se aplicar a lei de Snell-Descartes:

$n_{\text {sol }}=\frac{\operatorname{sen}\left(\theta_{\text {ext }}\right)}{\operatorname{sen}\left(\theta_{\text {int }}\right)}$

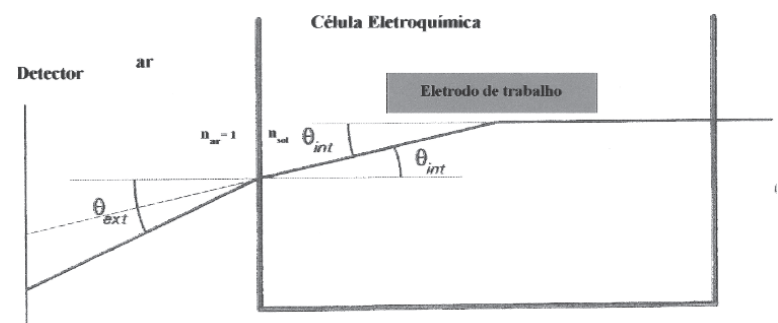

Figura 7. Desvio suplementar do feixe associado à sua passagem do interior para o exterior da célula eletroquímica. $O$ índice de refração da solução é maior que o índice de refração do $\operatorname{ar}\left(n_{\text {sol }}>1\right)$

Considerando que os ângulos $\theta_{\text {ext }}$ e $\theta_{\text {int }}$ são pequenos, os senos podem ser aproximados com seus ângulos:

$\theta_{\text {int }}=\frac{\theta_{e x t}}{n_{\text {sol }}}$

\section{LIMITAÇÕES DA MONTAGEM DO EFEITO MIRAGEM DE CONCENTRAÇÃO}

Tamanho do feixe laser no detector e distância entre as áreas ativas da foto-célula

A Figura 8 (a) e (b) representa o esquema de deslocamento do feixe laser que ilumina o detector e sua curva de calibração teórica associada, respectivamente.
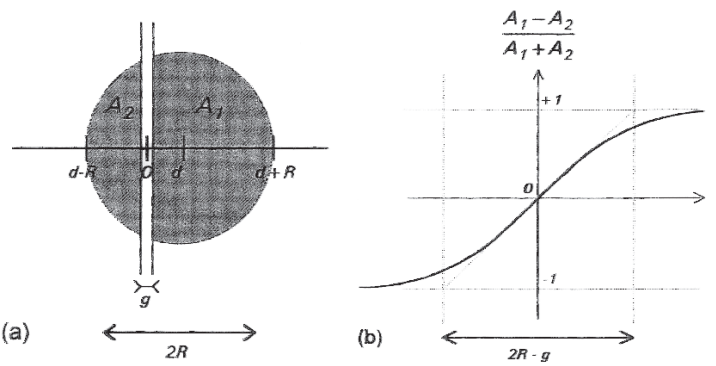

Figura 8. Deslocamento do feixe laser que ilumina o detector: (a) deslocamento do feixe que ilumina as duas áreas ativas da foto-célula; (b) curva de calibração teórica associada: $A_{1}$ e $A_{2}$ correspondem às superfícies iluminadas, $R$ é o diâmetro do feixe laser que ilumina a foto-célula, $d o$ deslocamento do feixe em relação ao centro do detector e $g$ a distância entre as áreas ativas da foto-célula

As superfícies $A_{1}$ e $A_{2}$ estão associadas com o deslocamento d do feixe pelas seguintes relações ${ }^{74}$ :

$$
\begin{aligned}
& A_{1}=R^{2}\left(\arccos (u)-u \sqrt{1-u^{2}}\right) \\
& A_{2}=R^{2}\left(\pi-\arccos (v)+v \sqrt{1-v^{2}}\right) \\
& \text { onde } u=\frac{-d+\frac{g}{2}}{R} \text { e } v=\frac{-d-\frac{g}{2}}{R}
\end{aligned}
$$

A partir das relações (9) e (10) é possível traçar a curva de calibração téorica (Figura 8b), onde está representada a expressão 
$\frac{A_{1}-A_{2}}{A_{1}+A_{2}}$ em função do deslocamento d. A relação entre o diâme tro (R) e a distância (g) é um aspecto importante para a definição da faixa de linearidade da curva de calibração, uma vez que a faixa de linearidade aumentará com a razão $\mathrm{R} / \mathrm{g}$.

\section{Diâmetro do feixe laser no ponto focal da lente}

A relação que permite calcular o diâmetro mínimo de um feixe focalizado por uma lente, levando-se em conta a repartição gaussiana da intensidade, é dada pela Equação ${ }^{74}$ :

$\phi=1,27 \frac{f}{D} \lambda$

sendo $f$ a distância focal da lente, $D$ o diâmetro inicial do feixe e $\lambda$ o comprimento de onda do feixe. Nossa montagem prevê um diâmetro da ordem de $32 \mu \mathrm{m}$.

Distância mínima, $x_{\text {minsd }}$, entre feixe laser e eletrodo de trabalho quando não existe desvio do feixe

A Figura 9 mostra a distância mínima de aproximação entre feixe laser e eletrodo de trabalho. Pode-se perceber que é impossível uma aproximação do eletrodo de trabalho inferior a uma distância mínima $\mathrm{x}_{\text {minsd }}$, visto que o diâmetro do feixe laser aumenta após o feixe passar pelo ponto focal da lente.

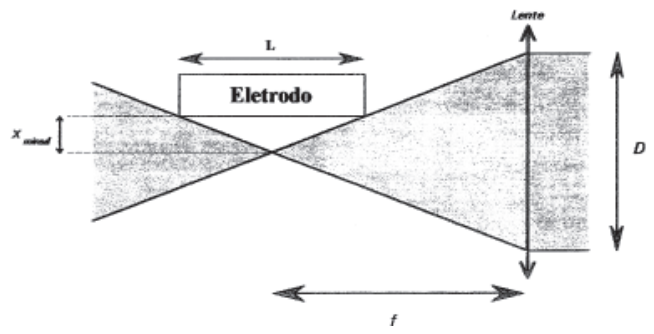

Figura 9. Distância mínima de aproximação entre feixe laser e o eletrodo de trabalho, onde $D$ é o diâmetro do feixe laser, $f$ a distância focal da lente, Lo comprimento do eletrodo e $x_{\text {minsd }}$ a distância mínima entre o feixe laser $e$ eletrodo de trabalho

Aplicando o teorema de Tales é possível estimar a distância mínima de aproximação:

$x_{\text {min sd }}=\frac{L D}{4 f}$

A partir dos parâmetros da montagem descrita na Figura 5 estimamos $\mathrm{x}_{\text {minsd }}=31,25 \mu \mathrm{m}$.

Distância mínima, $x_{\text {mincd }}$, entre o feixe laser e o eletrodo de trabalho quando existe desvio do feixe

Quando existe desvio do feixe a distância mínima determinada anteriormente deverá ser acrescentada de um termo $\mathrm{d}_{\mathrm{q}}$ correspondente ao deslocamento do feixe. Considerando as relações (5) e (12), a distância mínima será expressa por:

$$
x_{\min c d} \approx x_{\min s d}+\frac{L}{2} \theta \approx \frac{L}{2}\left(\frac{D}{2 F}+\theta\right)
$$

Caso, por ex., $\theta=5 \mathrm{mrad}$ (valor relativamente alto para nossos experimentos) e utilizando os parâmetros da montagem descrita na Figura 5 estimamos $x_{\text {mincd }}=43,75 \mu \mathrm{m}$.

\section{EXEMPLO DE UTILIZAÇÃO DO EFEITO MIRAGEM DE CONCENTRAÇÃO}

No intuito de ilustrar a técnica EM de concentração acoplada com a técnica de voltametria cíclica será apresentado, a seguir, um estudo que está sendo realizado em nosso laboratório, com o objetivo inicial de investigar qualitativamente os movimentos iônicos ocorridos durante a oxidorredução de um eletrodo de aço inoxidável AISI 304 de composição rica em cromo e baixa em carbono ${ }^{75}$. Os átomos presentes na composição, em $\% \mathrm{~m} / \mathrm{m}$, são $\mathrm{Cr}=18-20$, $\mathrm{Ni}=8-10,5, \mathrm{Si}=1,0, \mathrm{Mn}=2,0, \mathrm{~S}=0,03, \mathrm{C}=0,08, \mathrm{P}=0,045$, sendo estes átomos complementados por $\mathrm{Fe}$.

O aço inoxidável AISI 304 tem a propriedade, como algumas ligas de cromo ou alumínio, de passivar no ar. Este comportamento é devido a uma fina película aderente de óxido na superfície metálica ou a um estado oxidado da superfície. Quando um eletrodo de aço inoxidável é mergulhado em um meio corrosivo, como por ex., uma solução de $\mathrm{NaCl} 3 \% \mathrm{~m} / \mathrm{m}$, a camada de passivação pode ser destruída em pontos ou áreas localizadas (durante uma polarização anódica) a partir de um potencial, denominado potencial de pite. A ruptura localizada do filme passivo é devida, principalmente, aos íons cloretos que penetram na camada de óxido e aumentam sua permeabilidade ${ }^{76}$.

\section{Parte experimental}

O estudo EM de concentração foi realizado com o equipamento descrito na Figura 4. A célula eletroquímica utilizada é constituída de uma cubeta de quartzo de $50 \mathrm{~mm}$ de caminho ótico da NSG Precision Cells, contendo três eletrodos: o de trabalho é uma placa de aço inoxidável AISI 304 (área ativa $2 \mathrm{~cm}$ x $5 \mathrm{~mm}$ x $1 \mathrm{~mm}$ ) polida mecanicamente com lixas (mesh) 100, 220, 400, 600, 1000, 2000, 6, 3 e $1 \mu \mathrm{m}$ e limpa com acetona; o auxiliar é uma placa de platina (área ativa $2 \mathrm{~cm} \mathrm{x} 1 \mathrm{~cm}$ x $1 \mathrm{~mm}$ ) e o de referência é um eletrodo $\mathrm{Ag} / \mathrm{AgCl}$. A solução eletrolítica usada foi $\mathrm{NaCl} 3 \% \mathrm{~m} / \mathrm{m}$. A voltametria foi realizada utilizando um potenciostato/galvanostato digital Omnimetra modelo PG-39, na faixa de potencial de $-0,4$ até $+1,0 \mathrm{~V}$ com uma velocidade de varredura de $50 \mathrm{mV} \mathrm{s}^{-1}$. O detector de posição é constituído por uma foto-célula fotovoltáica de silício (Optilas, modelo SPOT-2DMI) que possui duas áreas ativas quadradas de $1,41 \mathrm{~mm}^{2}$, separadas por $10 \mu \mathrm{m}$.

\section{RESULTADOS E DISCUSSÃO}

Devido à pequena potência térmica associada à reação eletroquímica estudada não será considerada a participação do efeito térmico no desvio do feixe laser ${ }^{45}$.

A Figura 10 mostra a voltametria cíclica e o sinal miragem associado, obtidos durante o estudo do eletrodo de aço inoxidável AISI 304 na solução aquosa de $\mathrm{NaCl} 3 \% \mathrm{~m} / \mathrm{m}$.

Pode-se observar que, durante a varredura anódica (Figura 10a), a oxidação do aço inicia somente a partir de $+0,7 \mathrm{~V}$ (que representa o potencial de pite do eletrodo). A partir deste potencial, regiões localizadas na camada de passivação são destruídas e o aço começa a ser oxidado. $\mathrm{O}$ sinal miragem associado à varredura de oxidação (Figura 10b) não mostra nenhum movimento majoritário de íons. Em razão do movimento dos íons cloreto, que penetram na camada de óxido, não estar perceptível no sinal miragem podemos considerar que o transporte de íons cloreto é realizado principalmente por migração $0^{48}$ A ausência de desvio do feixe após $0,70 \mathrm{~V}$ 


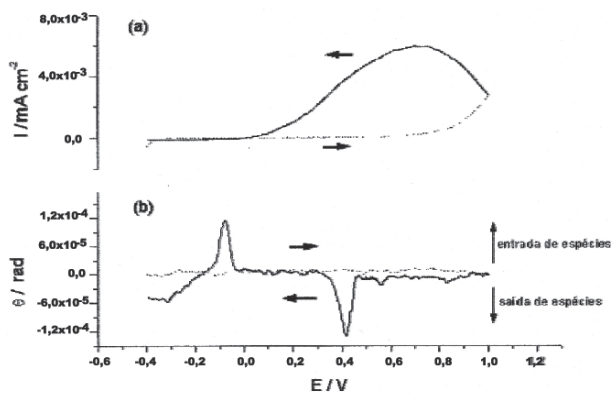

Figura 10. Voltametria cíclica e o sinal miragem associado, obtidos para o aço inoxidável AISI 304. (a) Voltamograma cíclico obtido com o eletrodo de aço inoxidável AISI 304 na solução aquosa de $\mathrm{NaCl} 3 \% \mathrm{~m} / \mathrm{v}$ : varredura de ida (—) e de volta (-). (b) Sinal miragem associado à varredura de ida (-) e de volta (-)

pode ser explicada pelo atraso na detecção de perturbação do perfil de concentração do eletrólito ${ }^{49}$.

Com o desaparecimento da camada de óxido, o aço continua a ser oxidado durante a varredura catódica, mesmo com a diminuição do potencial (Figura 10a). O sinal miragem associado à varredura de redução (Figura 10b) mostra dois picos. O primeiro indica um movimento majoritário de espécies do eletrodo em direção à solução (saída), que podemos associar principalmente à oxidação do ferro (dissolução). O segundo indica um movimento majoritário de espécies da solução em direção ao eletrodo (entrada), que podemos associar principalmente ao deslocamento de cátions $\left(\mathrm{Na}^{+}\right.$ e/ou $\mathrm{Fe}^{2+}$ ). A ausência de uma corrente negativa durante a redução (Figura 10a) mostra que, na faixa de potencial do experimento, os íons $\mathrm{Fe}^{2+}$ não são reduzidos (deposição) no eletrodo. No final da varredura de redução, abaixo de $-0,10 \mathrm{~V}$, o sinal miragem evidencia que existe um deslocamento predominante de espécies em direção à solução utilizada.

Este estudo encontra-se ainda em fase inicial. Estamos atualmente trabalhando com dois objetivos: acoplar o equipamento EM com uma MECQ e utilizar a convolução temporal para quantificar os processos iônicos que acontecem durante a oxidorredução de um eletrodo de aço inoxidável AISI 304.

\section{COMENTÁRIOS FINAIS}

A técnica EM, relacionada tanto com o gradiente de concentração quanto com o de temperatura, tem recebido grande atenção, particularmente como consequiência de sua simplicidade e sensibilidade.

$\mathrm{Na}$ área da eletroquímica, o EM de concentração permite uma interpretação alternativa para os processos físicos e químicos existentes nas reações e sua principal vantagem é a monitoração in situ da evolução dos fluxos de massa durante qualquer perturbação eletroquímica (voltametria cíclica, cronoamperometria, cronopotenciometria, etc.) aplicada no eletrodo de estudo. O acompanhamento das reações eletroquímicas por meio de uma técnica ótica que registra a mudança no índice de refração do eletrólito, utilizando um feixe de laser, possibilita uma visão seletiva dos efeitos iônicos resultantes do desequilíbrio presente na interface eletrodo/eletrólito, provocado pelos processos que ocorrem no eletrodo.

As dificuldades encontradas na obtenção de informações quantitativas a partir da técnica miragem são superadas, utilizando um tratamento matemático baseado na convolução temporal ou a partir de simulações digitais, permitindo a compensação dos atrasos que existem entre o sinal eletroquímico e o sinal miragem. Esses atrasos estão relacionados com o fato de que o gradiente de concentração detectado estará sempre situado a uma certa distância do eletrodo de trabalho, já que o feixe laser empregado na técnica EM não é pontual. Mesmo focalizado, ele possui um diâmetro mínimo de 30 a 70 $\mu \mathrm{m}$ e qualquer perturbação eletroquímica realizada neste eletrodo será detectada somente após um certo tempo pelo feixe laser.

Quando a reação eletroquímica estudada apresenta importantes trocas de calor (efeitos Peltier e Joule), é necessário separar os componentes de temperatura e de concentração do sinal miragem. Na maioria dos casos, os coeficientes de difusão térmicos são sensivelmente superiores aos coeficientes de difusão iônica e o sinal miragem térmico será detectado antes do sinal miragem de concentração.

A técnica EM de concentração não é sensível aos movimentos do solvente, logo, é interessante acoplar o EM a outras técnicas eletroquímicas, como a MECQ, a fim de se obterem maiores informações sobre os transportes de massas que ocorrem durante os processos eletroquímicos estudados.

Alguns trabalhos publicados recentemente mostraram aplicações atuais da técnica EM de concentração em diferentes áreas. Brolo et $a l .{ }^{77}$ investigaram um complexo mecanismo de formação de filmes de $\mathrm{AgBr}(\mathrm{s})$ sobre eletrodo de prata e mostraram a complementaridade do EM com as técnicas eletroquímicas clássicas. Correia et al. ${ }^{51}$ empregaram a técnica EM para estudar os mecanismos redox de polímeros condutores. Bidoia ${ }^{78}$ utilizou o EM para estudar a adsorção de ânions $\left(\mathrm{H}_{2} \mathrm{PO}_{4}^{-}\right)$em eletrodos de platina, mostrando que a técnica permite monitorar a interface platina/eletrólito com um custo bem inferior à Espectroscopia de Infravermelho com Transformada de Fourrier (FTIR), técnica habitualmente empregada neste tipo de estudo. Barbero ${ }^{79}$ mostrou os interesses da utilização da técnica EM no estudo das troca iônicas que existem em vários sistemas, como os polímeros condutores e redox, carregamento da dupla camada dos carbonos nanoestrurados etc. A técnica tem se apresentado como uma ferramenta apropriada para estudar os efeitos do tamanho dos íons, do $\mathrm{pH}$, do solvente, da composição dos filmes, da velocidade dos íons etc.

Finalmente, esperamos que este trabalho contribua para a divulgação e difusão da técnica em vários laboratórios brasileiros.

\section{AGRADECIMENTOS}

Ao apoio da Fundação de Amparo à Ciência do Estado de Minas Gerais (FAPEMIG) e do Conselho Nacional de Desenvolvimento Científico e Tecnológico (CNPq).

\section{REFERÊNCIAS}

1. Varela, H.; Malta, M.; Torresi, R. M.; Quim. Nova 2000, 23, 664.

2. Barbero, C.; Miras, C.; Kotz, R.; Electrochim. Acta 1992, 37, 429.

3. Muller, R. H.; Advances in Electrochemistry and Electrochemical Engineering, John Wiley \& Sons: New York, 1973.

4. McLarnon, F. R.; Muller, R. H.; Tobias, C. W.; J. Electrochem. Soc. 1975, $122,59$.

5. McLarnon, F. R.; Muller, R. H.; Tobias, C. W.; Electrochim. Acta 1976, 21,101.

6. McLarnon, F. R.; Muller, R. H.; Tobias, C. W.; Ind. Eng. Chem. Fun. 1979, 18, 97.

7. Rudnicki, J. D.; McLamon, F. R.; Caims, E. J. Em Techniques for Characterization of Electrodes and Electrochemical Processes; Varma, R.; Selman, J. R., eds.; Plenum: New York, 1991.

8. Barbero, C.; Miras, C. Em Tecnicas deflectometricas aplicadas al studio de procesos electroquimicos; Alonso-Vante, N., ed.; E-libro net: Buenos Aires, 2003

9. Boccara, A. C.; Fournier, D.; Badoz, J.; Appl. Phys. Lett. 1980, 36, 130.

10. Russo, R. E.; McLarnon, F. R.; Spear, J. D.; Cairns, E. J.; J. Electrochem. Soc. 1987, 134, 2783.

11. Decker, F.; Neuenschwander, R. T.; Cesar, C. L.; Fenna, A. F. S.; J. Electroanal. Chem. 1987, 228, 481. 
12. Jackson, W. D.; Amer, N. M.; Boccara, A. C.; Fournier, D.; Appl. Opt. 1981, 20, 1333.

13. Roger, J. P.; Fournier, D.; Boccara, A. C.; Noufi, R.; Cahen, D.; Thin Solid Films 1985, 128, 11.

14. Vieil, E.; Lopez, C.; J. Electroanal. Chem. 1999, 466, 218.

15. Matencio, T.; Vieil, E.; Synth. Met. 1991, 44, 349.

16. Decker, F.; Fracastoro-Decker, M.; J. Electroanal. Chem. 1988, 243, 187.

17. Vorotyntsev, M. A.; Lopez, C.; Vieil, E.; J. Electroanal. Chem. 1994, 368, 155.

18. Walther, H. G.; J. Appl. Phys. 2000, 98, 122.

19. Salloum, A.; Atassi, Y.; Thermochim. Acta 2004, 409, 87.

20. Rousset, G.; Lepoutre, F.; Rev. Phys. Appl. 1982, 17, 201.

21. Inglehart, L. J.; Lepoutre, F.; Charbonnier, F.; J. Appl. Phys. 1986, 59, 234

22. Shannon, M. A.; Rubinsky, B.; Russo, R. E.; J. Appl. Phys. 1994, 75, 1473.

23. Wu, X. Z.; Shindoh, H.; Yamada, M.; Hobo, T.; Anal. Chem. 1993, 85, 834.

24. Wu, X. Z.; Hobo, T.; Anal. Chim. Acta 1995, 316, 111.

25. Schneider, T.; Politi, M. J.; Baptista, M. S.; Thermochim. Acta 2000, 362, 179 .

26. Wu, X. Z.; Morikawa, T.; Uchiyama, K.; Hobo, T.; J. Phys. Chem. B 1997, 101,1520 .

27. Kuo, P. K.; Lin, M. J.; Reyes, C. B.; Favro, L. D.; Thomas, R. L.; Kim, D. S.; Zhang, S.; Inglehart, L. J.; Fournier, D.; Boccara, A. C.; Can. J. Phys. 1991, 69, 1216.

28. Kuo, P. K.; Sendler, E. D.; Favro, L. D.; Thomas, R. L.; Can. J. Phys. 1986, 64, 1168

29. Salazar, A.; Sánchez-Lavega, A.; Fernández, J.; J. Appl. Phys. 1991, 69, 1216

30. Bialkowski, S. E.; Photothermal Spectroscopy Methods for Chemical Analysis, Wiley: New York, 1996.

31. Krapez, J. C.; Ing. Surf. 2001, 26, 328.

32. Sun, J.; Longtin, J. P.; Irvine, T. F.; Int. J. Heat Mass Transfer 2001, 44, 645 .

33. Boubaker, K. M.; Bouhafs, M.; Yacoubi, N.; NDT \& E Int 2003, $36,547$.

34. Lucia, U.; Maino, G.; Nucl. Instrum. Methods Phys. Res., Sect. B 2004, $213,139$.

35. Liu, X. J.; Huang, Q. J.; Zhang, S. Y.; Luo, A. H.; Zhao, C. X.; J. Phys. Chem. Solids 2004, 65, 1247.

36. Petrovsky, A. N.; Salnick, A. O.; Zuev, V. V.; Mukhin, D. O.; Mekhtiev, M. M.; Pelzl, J.; Boccara, A. C.; Fournier, D.; Solid State Commun. 1992, 81, 223.

37. Mathew, A.; Ravi, J.; Madhusoodanan, K. N; Nair, K. P. R.; Rasheed, T. M. A.; Appl. Surf. Sci. 2004, 227, 410.

38. Dersch, H.; Amer, N. M.; Appl. Phys. Lett. 1984, 45, 272.

39. Tu, C. Y.; Kitamori, T.; Sawada, T.; Kimura, H.; Matsuzawa, S.; Anal. Chem. 1993, 65, 3631.

40. Havaux, M.; Lorrain, L.; Leblanc, M.; FEBS Lett. 1989, 250, 395.

41. Lorrain, L.; Havaux, M.; Tessier, A.; Leblanc, M.; Photochem. Photobiol. Sci. 1990, 51, 491.

42. Murphy, J. C.; Aamodt, L. C.; J. Appl. Phys. 1980, 51, 4580.

43. Mandelis, A.; J. Appl. Phys. 1983, 54, 3404.

44. Bidoia, E. D.; McLarnon, F.; Cairns, E. J.; J. Electroanal. Chem. 2000 $482,75$.

45. Rosolen, J. M.; Fracastoro-Decker, M.; Decker, F.; J. Electroanal. Chem. 1993, 346, 119 .
46. Fracastoro-Decker, M.; Decker, F.; J. Electroanal. Chem. 1989, 266, 215.

47. Rudnicki, J. D.; Brisard, G. M.; Gasteiger, H. A.; Russo, R. E.; McLarnon, F. R.; Cairns, E. J.; J. Electroanal. Chem. 1993, 362, 55.

48. Mathias, M. F.; J. Electroanal. Chem. 1996, 407, 115.

49. Vieil, E.; Meerholz, K.; Matencio, T.; Heinze, J.; J. Electroanal. Chem. 1994, 368, 183.

50. Vieil, E.; J. Electroanal. Chem. 1994, 264, 9.

51. Correia, J. P.; Vieil, E.; Abrantes, L. M.; J. Electroanal. Chem. 2004, 573, 299.

52. Roger, J. P.; Fournier, D.; Boccara, A. C.; J. Phys. (Paris), Colloq. C6. 1983, 44, 313.

53. Matencio, T.; De Paoli, M. A.; Peres, R. C. D.; Torresi, R. M.; Torresi, S. I. C.; Synth. Met. 1995, 72, 59.

54. Royce, B. S. H.; Voss, D.; Bocarsly, A.; J. Phys. (Paris), Colloq. C6. 1983, 44, 325

55. Plinchon, V.; Besbes, S.; J. Electroanal. Chem. 1990, 284, 141.

56. Rosolen, J. M.; Decker, M. F.; Decker, F.; J. Electroanal. Chem. 1994, 365, 165 .

57. Matencio, T.; Peres, R. C. D.; Torresi, R. M.; Torresi, S. I. C.; De Paoli, M. A.; J. Braz. Chem. Soc. 1994, 5, 191.

58. Csahók, E.; Vieil, E.; Inzelt, G.; J. Electroanal. Chem. 1998, 457, 251.

59. Levi, M. D.; Levi, E.; Gofer, Y.; Aurbach, D.; Vieil, E.; Serose, J.; J. Phys. Chem. B 1999, 103, 1499.

60. Stefan, I. C.; Tolmachev, Y. V.; Scherson, D. A.; Anal. Chem. 2001, 73, 527.

61. French, H. M.; Henderson, M. J.; Hillman, A. R.; Vieil, E.; Solid State Ionics 2002, 150, 27.

62. Brolo, A. G.; Sharma, S. D.; Electrochim. Acta 2003, 48, 1375.

63. Girotto, E, M.; De Paoli, M. A.; Quim. Nova 1999, 22, 358.

64. Henderson, M. J.; Hillman, A. R.; Vieil, E.; J. Electroanal. Chem. 1998, 454,1 .

65. Vilas-Boas, M.; Henderson, M. J.; Freire, C.; Hillman, A. R.; Vieil, E.; J. Chem. Eur. 2000, 6, 1160 .

66. Henderson, M. J.; Hillman, A. R.; Vieil, E.; J. Phys. Chem. B 1999, 103, 8899.

67. Henderson, M. J.; French, H.; Hillman, A. R.; Vieil, E.; Electrochem. SolidState Lett. 1999, 2, 631.

68. Trigueiro, J. P. C.; Matencio, T.; Resumos da $27^{a}$ Reunião Anual da Sociedade Brasileira de Química, Salvador, Brasil, 2004.

69. Matencio, T.; XIV Simpósio Brasileiro de Eletroquímica e Eletroanalítica, Teresópolis, Brasil, 2004.

70. Svelto, O.; Hanna, D.; Principles of Lasers, $4^{\text {th }}$ ed., Plenum Press: New York, 1998.

71. Andrews, D., L.; Lasers in Chemistry, $3^{\text {rd }}$ ed., Springer: Berlin, 1997.

72. Erickson, J. O.; Surles, T.; Am. Lab. 1976, 8, 50.

73. Oliveira, E. A.; Dos Santos, P. A. M.; Cescato, L.; Mendes, G. F.; Frejlich, J.; Rev. Fis. Apl. Inst. 1985, 1, 1.

74. Lopez, C.; Tese de Doutorado, Université Joseph Fourier, France, 1994.

75. Moraga, G. A.; Tese de Doutorado, Universidade Federal de Minas Gerais, Brasil, 2005.

76. Gentil, V.; Corrosão, $3^{\text {a }}$ ed., LTC: Rio de Janeiro, 1998.

77. Brolo, A. G.; Yang, Y.; Electrochim. Acta 2004, 49, 339

78. Bidoia, E. D.; Chem. Phys. Lett. 2005, 408, 1.

79. Barbero, C.; Phys. Chem. Chem. Phys. 2005, 7, 1885 